\title{
EDITORIAL
}

\section{Investigar en educación artística: nuevos entornos y retos pendientes}

\author{
Research in arts education: new environments and challenges ahead
}

Ricard Huerta. Universitat de València. ricard.huerta@uv.es

Ricardo Domínguez. Universitat de València. ricardo.dominguez@uv.es

\section{A Elliot W. Eisner. In memoriam}

Resumen: Investigar en educación artística nunca fue fácil, y ciertamente las dificultades son ahora mayores. Si bien se ha podido avanzar en muchos sentidos, lo cierto es que en la actualidad no disponemos de mecanismos suficientes para desarrollar tareas de amplio calado, tanto a nivel curricular como en lo referido a consolidar la presencia social o mediática. En tanto que área de conocimiento estamos afrontando una situación que resulta cada vez más precaria, y debemos tomar conciencia de la gravedad de esta circunstancia. La creación de grupos interdisciplinares y la apertura hacia nuevas zonas de acción suponen esfuerzos que conviene abordar de forma coordinada. La necesidad de internacionalizar las miradas y las acciones supone encontrar aliados tanto en otras geografías como en diferentes ámbitos de maniobra. Sabemos y asumimos que se ha avanzado, pero ante todo debemos arriesgar de cara al futuro, con el fin de propiciar nuevos entornos y abrir espacios de reflexión, conscientes de nuestras posibilidades. Revisamos aquí algunos de los logros conseguidos y desarrollamos nuevas propuestas de intervención.

Palabras clave: educación artística, investigación, arte, formación de docentes.

Abstract: Investigate in art education was never easy, and certainly the difficulties are greater now. It has made progress in many ways, but currently do not have sufficient mechanisms to develop new tasks. We note that our presence is decreasing in the curriculum, and we have little presence on social or media level. Our area of 
expertise is facing a situation that is increasingly precarious, and we must be aware of the seriousness of this. The creation of interdisciplinary groups is essential. Openness to new areas of action has to implement a series of efforts that should be addressed with greater coordination. We need to internationalize the actions to find allies both in other geographies and in different policy areas. We must be aware of everything that has been achieved, but above all we must risk the future, in order to encourage new environments and open spaces for reflection, aware of our possibilities. We review here some of the achievements and develop new intervention proposals.

Keywords: art education, research, art, training teachers.

\section{Introducción}

En el año 2003 aparecía el primer número de la revista EARI (www.revistaeari. org) cuyo monográfico se titulaba "Radiografía de la Educación Artística". El nombre presagiaba algo de diagnóstico, referido a una situación que podríamos definir como delicada. Ya entonces justificábamos que uno de los motivos principales al crear nuestra publicación radicaba en editar de forma regular las opiniones e investigaciones de los especialistas en educación artística, animando así al colectivo a participar en encuentros periódicos para generar debates sobre las diversas temáticas que pudiesen interesar. Si revisamos lo que ha ocurrido en el área durante los últimos 15 años observamos que se ha avanzado mucho, tanto en la celebración de seminarios, congresos y reuniones, como en la edición de libros y en la publicación de artículos especializados. También se han creado grupos de investigación que están desarrollando proyectos muy variados e interesantes. Pero a pesar de todo ello, el espectro curricular de la educación artística continúa cayendo en picado tanto en la educación primaria, como en secundaria y en la universidad, al tiempo que nuestra presencia como área especializada sigue sin contar con la visibilidad suficiente ni en el ámbito de lo político, ni en lo social, ni tampoco en el espacio mediático. Seguimos sin disponer de referencias poderosas, y el hecho de haber perdido este año a Elliot W. Eisner, figura clave durante la segunda mitad del siglo XX, delata una cierta situación de fragilidad, ya que las personalidades que sirven para orientarse resultan siempre un fuerte apoyo de cara a conseguir integrar a los colectivos con intereses peculiares. A pesar de las dificultades, reivindicamos una mayor adaptación de la educación artística en todas las instancias, seguimos luchando para que el arte y la educación encuentren caminos de concertación, y desde luego animamos a los profesionales de nuestra área a reflexionar sobre las posibilidades futuras y a gestionar del mejor modo posible sus acciones. Compartiendo estas ilusiones estaremos en condiciones de superar los retos que se nos plantean. 


\section{La importancia de las organizaciones internacionales y de las redes de comunicación}

Pronto se cumplirán seis décadas desde la fundación de InSEA, la International Society for Education through Art (www.insea.org), una organización vinculada a UNESCO (www.unesco.org), y una entidad clave para el fomento de la educación artística desde una perspectiva mundial. El papel de Herbert Read en la fundación de InSEA fue clave, y las relecturas de su libro Education through Art (Read, 1982), escrito originalmente en la década de 1940, siguen dando pie a numerosas reflexiones. Del mismo modo podríamos recordar el trabajo de personalidades que presidieron en su momento InSEA como puedan ser el propio Elliot W. Eisner, la inquebrantable Ana Mae Barbosa, o recientemente Rita Irwin, quien ha lidiado el fuerte debate por mantener el papel esencial de la educación artística entre las prioridades de UNESCO. Las reuniones de InSEA están muy pendientes de la realidad de cada momento histórico, como es el caso del congreso mundial celebrado este año en Melbourne bajo la temática general de la diversidad (www.insea2014. com). Queremos destacar aquí la importancia de algunas organizaciones nacionales que constituyen la red InSEA, entre las que encontramos las norteamericanas NAEA (www.arteducators.org) e INAEA (www.inaea.org), la británica (www. nsead.org), o la más cercana a nosotros que sería la portuguesa APECV (www. apecv.pt). Precisamente de Portugal es la actual presidenta de InSEA Teresa Torres de Eça, quien ha promovido también redes de contacto tan interesantes como RIAEA (https://groups.yahoo.com/neo/groups/riaea/info), un entorno online que constituye en estos momentos una buena plataforma para integrar y potenciar lo que estamos haciendo en el ámbito iberoamericano. Consideramos fundamental que se fortalezcan este tipo de organizaciones, animando al profesorado y a los investigadores en educación artística a implicarse directamente como asociados y gestores en estas redes internacionales.

Las redes sociales y el entorno online han permitido fortalecer los contactos, uniendo a investigadores de diferentes países y estableciendo pautas de cooperación que están abriendo novedosas expectativas de trabajo. Los problemas son comunes, y un factor como la crisis económica permite impulsar algunos retos que antes no parecían importantes. En países como India, la utilización del potencial de la educación artística para fomentar el emprendimiento resulta muy interesante $\mathrm{y}$ atractivo, ya que refleja un cambio de posicionamiento desde lo meramente expresivo hacia las cuestiones sociales y económicas, mucho más urgentes y palpables en los países emergentes como Brasil, India o China. Desde esta potencia y desde el resto de geografías asiáticas empiezan a escucharse nuevas voces, con una fuerza inusitada, y la presencia de representantes de China, Taiwán o Polinesia en los foros internacionales resulta cada día más significativa. Podemos afirmar que es la situación global la que está moviendo en realidad el mecanismo de la educación artística. Para nosotros, generadores de imágenes y estudiosos de sus capacidades, este nuevo reto supone iniciar los contactos con grupos de las más 
diversas nacionalidades. Puede que en el pasado tuviésemos un cariz marcadamente occidental, pero el futuro tiene muchas caras nuevas, y la diversidad está en la base de esta riqueza.

\section{Revistas de referencia y otras publicaciones sobre educación artística}

Desde que EARI está disponible online, hemos detectado que, tal y como era previsible, el espectro de lectores de la revista abraza mayormente el ámbito iberoamericano, al tiempo que detectamos un buen número de especialistas estadounidenses interesados por nuestros artículos. El hecho de publicar en español confiere a la revista una personalidad propia, pero ante todo permite a nuestro público, tanto autores como lectores, expresarse en un lenguaje que nos sirve como estrategia comunicativa. El hecho de utilizar el OJS Open Journal System como plataforma de edición y difusión es la clave para indexar la publicación, que ya está indizada en catálogos como Latindex, Dialnet o Dulcinea, desde donde pensamos dar el paso hacia nuevas instancias. Otras publicaciones que son referencia en nuestro entorno son las revistas editadas por la Universidad Complutense, como es el caso de la prestigiosa y de larga trayectoria Arte, Individuo y Sociedad (www. arteindividuoysociedad.es) o bien la más reciente pero ya consolidada Arteterapia. Papeles de arteterapia y educación artística para la inclusión social (http://revistas. ucm.es/index.php/ARTE). El impulso que se ha dado desde Portugal a nuestra área, siempre con el saber hacer que vierten profesionales como Teresa Torres o Ricardo Reis, y tanta personas entusiastas que se organizan profesionalmente en torno a APECV, ha permitido generar e impulsar publicaciones tan atractivas como InVisibilidades (http://invisibilidades.apecv.pt/ojs) o Imaginar (http://apecv. $\mathrm{pt} / \mathrm{q}=$ node/14). No pretendemos ahora hacer un balance exhaustivo, algo que necesita ser recopilado con urgencia, pero sí queremos demostrar que el papel de estas publicaciones resultará esencial en las derivas que pueda tomar la educación artística, ya que los entornos de investigación en las áreas de ciencias sociales y de humanidades han de contar con publicaciones especializadas que alcancen la mayor difusión y que doten de prestigio al conjunto de nuestros trabajos. Algunos grupos de investigadores de Uruguay, Argentina, Chile, Perú, Colombia, México y muy especialmente Brasil, están iniciando tareas de edición que, con toda seguridad darán resultados positivos en el futuro. En el ámbito anglosajón, y gracias al empuje que toma el inglés como lengua dominante en el terreno de la investigación científica, son muchas las publicaciones a las cuales podemos acceder. Citaremos dos de las más interesantes, a saber, el muy bien situado International Journal of Art and Design Education (http://onlinelibrary.wiley.com/doi/10.1111/jade.2014.33. issue-2/issuetoc), o la pujante IJETA (http://ojs.library.ubc.ca/index.php/ijeta) que tiene vinculación directa con InSEA.

En el apartado de publicaciones de libros, cabe destacar al nuevo escenario que supone la edición online, que en el caso de las universidades nos permite el acceso 
libre y gratuito a este tipo de ediciones especializadas. Son los grupos de investigación quienes están impulsando esta nueva modalidad, lo cual supone un claro avance en lo referido a difusión de nuestras investigaciones. De nuevo comprobamos que es la internacionalización el mejor de los escenarios posibles, ya que no somos una población mayoritaria en cuanto a número de implicados, y hemos de ser conscientes de que otras áreas de conocimiento como las didácticas de la lengua, de las matemáticas, o de las ciencias sociales, tienen una mayor tradición y manejan otras cifras de implicados mucho mayores. El empuje que están tomando los blogs especializados, un espacio menos formal pero no menos enriquecedor que el propio de las publicaciones editadas, demuestra también la cantidad de profesorado que sigue atentamente la evolución de lo que está ocurriendo en educación artística.

\section{Reuniones científicas en las que se verifica el interés por la investigación en educación artística}

Ya nos hemos referido a la importancia de los congresos mundiales y regionales que se organizan desde InSEA, pero ciertamente son las universidades y las asociaciones profesionales quienes están impulsando numerosas reuniones, en las que se debaten temas de interés, y mediante las cuales se difunden los trabajos de los compañeros y las compañeras que están investigando en temáticas que resultan cada vez más abiertas e innovadoras. Debido a la cercanía y a un mayor conocimiento en directo de lo que ocurre, me referiré con algunos ejemplos a lo que está pasando en España y Portugal, dos países fuertemente afectados por las medidas económicas que se están tomando desde la Unión Europea. A pesar de las dificultades, seguimos aportando ideas y reforzando nuestro posicionamiento en el panorama de la investigación. El reto de la penuria económica nos lleva a tomar soluciones creativas, y especialmente a gestionar los entornos online, desde donde podemos además solucionar los problemas que suponen las distancias geográficas y los desplazamientos de las personas. La prueba es el éxito que ha tenido el reciente $2^{\circ}$ Congreso Internacional de RIAEA (http://2riaea26apecv.blogspot.com.es), o la convocatoria del Congreso Internacional Educación Artística y Diversidad Sexual (http://artemaestrosymuseos.wordpress.com), a celebrar en Valencia los días 13 y 14 de noviembre de 2014, y que permite poner en contacto a investigadores de numerosos países gracias a la opción de comunicaciones online, una modalidad mucho más accesible y sugerente. 


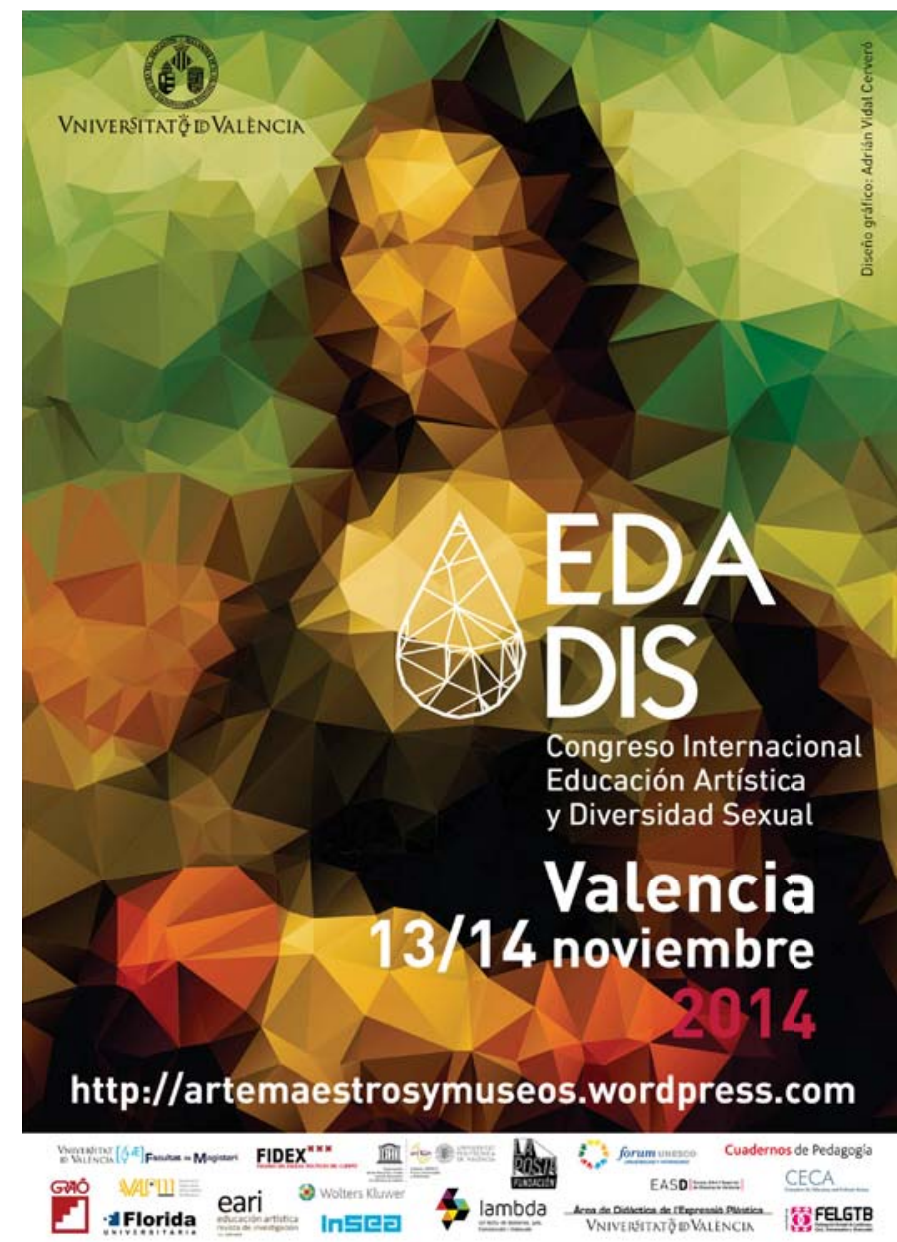

Fig 1. Cartel EDADIS

El tránsito de imágenes e ideas supone un fuerte respaldo a nuestro posicionamiento desde el entorno virtual. Por otro lado, el hecho de introducir temáticas de orden social, cultural y de género, como es el caso de la diversidad sexual, permitirá asimismo ampliar el espectro de las posibilidades de la educación artística. Pensamos que este nuevo enfoque, mucho más implicado social y políticamente, puede fortalecer nuestro discurso (Huerta, 2014a).

\section{Grupos de investigación y proyectos que se llevan a cabo}

Resulta motivador comprobar que ante el despropósito de las medidas que están suponiendo los recortes económicos, los cuales nos están afectando de manera negativa en investigación científica y en educación, sigue habiendo una predisposición por parte de los y las profesionales para avanzar en algunos logros conseguidos y mantener así un fuerte espíritu de lucha. Una plataforma como el grupo esbrina (www.ub.edu/esbrina), coordinado por Fernando Hernández, nos permite comprobar que en las cuestiones de la investigación educativa de carácter cualitativo todavía tenemos mucho terreno por recorrer. También resultan interesantes 
las propuestas que va definiendo Edarte (http://edarte.org), cuyos responsables son el tándem Imanol Aguirre y Amaia Arriaga, quienes están realizando un importante trabajo a partir de las producciones visuales de los jóvenes. No podemos perder de vista las acciones de un clásico como el MuPAI (http://mupai.wordpress.com), coordinados por Manuel Hernández Belver y María Acaso, y que constituyen un referente desde hace décadas. El interés por la educación patrimonial ha llevado a Olaia Fontal a dibujar un poderoso panorama que tiene como siglas OEPE (www. oepe.es), y que desde el magisterio de personalidades como Roser Calaf supone un importante avance para quienes nos interesamos por el patrimonio y la educación. La trayectoria dilatada de Ricardo Marín ha permitido a la Universidad de Granada generar numerosos encuentros y ediciones, del mismo modo que Roser Juanola desde la Universitat de Girona o María Jesús Agra desde la Universidade de Santiago de Compostela gestionan un fuerte potencial creativo en aspectos que van desde la educación patrimonial hasta las geografías expresivas. Los congresos organizados por la Universidad de Sevilla, la Universidad de Jaén y la Universidad de Málaga son otro de los alicientes que llegan desde Andalucía para configurar este panorama que esbozamos de forma rápida pero que resulta muy motivador.

\section{Cursos y masters de formación para el profesorado en educación artística}

Nunca antes el panorama universitario en la oferta de masters y de cursos de especialización había resultado tan generoso en relación con la educación artística. Tanto desde la visión de género (es el caso del organizado por Marián López Fdez.-Cao en la Complutense de Madrid), como desde la visión construccionista (en el que participan varias universidades de Andalucía y Cataluña), como desde el espectro de la educación patrimonial, el alumnado interesado por investigar estas cuestiones dispone ahora de múltiples opciones para elegir. En la Universitat de València hemos optado por integrar nuestra área de Didáctica de las Artes Visuales dentro del Master de Investigación en Didácticas Especificas (www. uv.es/masterdidacticas), y también disponemos de titulaciones específicos como es el caso del Diploma de Posgrado en Educación Artística y Gestión de Museos (http://postgrado.adeit-uv.es/14412070) con sus catorce ediciones consecutivas, o el recientemente ofertado Certificado en Arte, Tecnologías y Educación (http:// postgrado.adeit-uv.es/14414080). Precisamente el Diploma en Educación Artística y Gestión de Museos, que fue galardonado con el Premio Universidad-Empresa, ha sido el germen de la muy activa AVALEM, la Asociación Valenciana de Educadores de Museos y Patrimonios, una organización profesional que ha sabido instalarse dentro del panorama cultural de la ciudad, que colabora con museos, galerías y entidades, y que ya constituye por méritos propios un hito en esta tipología actual de plataformas organizativas (http://avalem.wordpress.com). 


\section{Nuevas metodologías para la investigación en educación artística y entornos propicios para la educación en artes}

Cuando inaugurábamos EARI en 2003 todavía nos bañábamos metodológicamente en las aguas del siglo anterior. Tras una década de cambios y adecuaciones podemos atestiguar que la variedad con que ahora afrontamos nuestras investigaciones resulta mucho más interesante, sin duda. Una de las claves de esta evolución está en la propuesta del VCAE (Visual Culture Art Education) que promulgó en su momento Paul Duncum, a partir de su inicial postulado de la Cultura Visual, incorporando posteriormente un guiño cómplice al DBAE (Discipline Based Art Education) de Elliot W. Eisner (Duncum, 2002). Con la premisa de la cultura visual y un acertado planteamiento estético desde la educación, Duncum sigue hoy en día incorporando matices a su propuesta, y desde luego ha favorecido muchas e interesantes actuaciones en lo referido a la apreciación de las producciones y los saberes que manejan los adolescentes, quienes disfrutan de una imaginería propia y particular. Desde otras latitudes nos llegaba la propuesta de las artografías, un recopilatorio de lo que habían sido las teorías del artist-teacher (Parker, 2009), pero en este caso incorporando factores relevantes como el espacio educativo o la importancia de la reflexión desde la investigación. Las siglas $\mathrm{A} / \mathrm{R} / \mathrm{T}$ se refieren a la tríada Artist/Researcher/Teacher, y con sus "a/r/tographies" Rita Irwin ha interpelado al educador, al artista y al investigador que cada docente llevamos dentro, al tiempo que defiende una mayor participación de los implicados en cada propuesta (especialmente el alumnado), insinuando el potencial que adquieren los escenarios en los que ejercemos la docencia (Irwin \& O’Donoghue, 2012). El hecho de que las artografías impulsen los trabajos desde la imagen, y que los fotoensayos estén dominando el panorama de la Educación Basada en las Artes (Arts Based Education), nos llevan hacia una nueva percepción de los trabajos de investigación que surgen desde la educación artística. Si bien es cierto que todavía se han de concretar muchos aspectos, también es verdad que ya empezamos a acostumbrarnos a recibir algunos TFG (trabajos finales de grado) o algunos TFM (trabajos finales de máster) en los cuales el alumnado opta por identificarse con la imagen, restringiendo así el poder magnánimo que hasta ahora mantenía el texto en el discurso propio de los investigadores y en las delimitaciones científicas de nuestras pautas de actuación. Puede que el escenario que más se ha afianzado en nuestros planteamientos sea el de la investigación cualitativa que proviene de la tradición antropológica y sociológica de los case studies (estudio de casos), que tanta repercusión han tenido en el ámbito educativo y en el universo de las artes (Huerta, 2014b). Este modelo metodológico permite oír las voces de muchos docentes que hasta ahora habían visto su trabajo relegado a la esclusa de la invisibilidad (Huerta, 2010). Lo cierto es que la investigación a partir de las llamadas Historias de Vida ha complementado muchos de los vacíos que resultaban excesivamente habituales en nuestro panorama investigador. Homenajear a los y las docentes que hacen su trabajo de forma prácticamente anónima es una manera de valorar una tradición que tenemos como colectivo. Investigar también supone remover las cuestiones que nos afectan. Llevamos años generando aperturas hacia nuevos escenarios (los 
museos, las galerías de arte, el entorno urbano, el universo online), y debemos tomar conciencia de que desde nuestras investigaciones podemos conseguir que la educación artística se convierta en un verdadero motor de cambio social (Alderoqui y Pedersoli, 2011; Augustowsky, 2012).

\section{En la encrucijada}

En el primer número de la revista EARI (2003) se realizaba una radiografía de la Educación Artística que fue premonitoria en relación sobre el poco valor que en general se le otorga a la misma y la tendencia progresiva a su desaparición de los currículos educativos. Desde esa percepción se reivindicaba la necesidad de esta publicación como espacio para compartir reflexiones, investigaciones y experiencias que aglutinaran esfuerzos y contribuyeran a la visibilidad de esta área de conocimiento.

En la segunda etapa de la revista, iniciada en el 2011, ésta se ha consolidado cumpliendo con su compromiso y periodicidad anual, incrementando su impacto mediante el acceso libre a sus contenidos a través de la web.

En este recorrido un total de 99 artículos dibujan un amplio territorio que nos permite acercarnos a cuestiones como: la educación patrimonial, la identidad, la educación artística y la cultura visual, la educación basada en las artes, la investigación educativa o la formación de docentes, por citar algunos (Fig. 2).

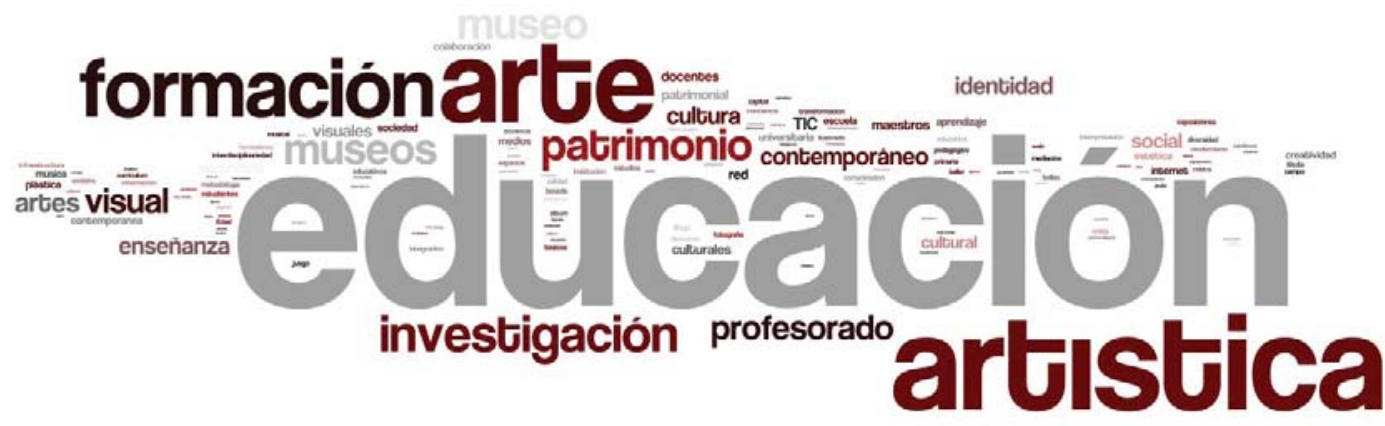

Fig. 2. Nube de tags a partir de los artículos publicados en la revista EARI.

Las pedagogías emergentes y el impacto de las tecnologías definen un nuevo escenario educativo en el que se diluyen las tradicionales fronteras entre ámbitos formales, no formales e informales; entre espacios físicos y virtuales; entre público y privado, o entre autor y espectador. En este contexto híbrido la Educación Artística es un fiel sismógrafo de los retos y necesidades de la sociedad actual. En este número se presentan 12 artículos en los que podemos ver algunas de estas cuestiones. 
En el ámbito de la formación de docentes situamos los siguientes artículos. En Dripping and Granering. A golpe de mancha. Una experiencia interdisciplinar en el ámbito de la educación universitaria Jose Rafel Adell y Lucía Rueda nos presentan una experiencia formativa desarrollada en la asignatura Educación Física, Plástica, Música y su Didáctica en las NEE, para el alumnado de $4^{\circ}$ de Grado de Educación Primaria en la especialidad de Pedagogía Terapéutica (PT). Desde el trabajo conjunto de un especialista en música y otro en artes plásticas plantea una experiencia formativa que incide en la interdisciplinariedad, el trabajo por proyectos y la cooperación.

El texto de Glòria Jové y Olga Olivera, Figuras homotéticas y otros diálogos con el brócoli romanesco. "Entre" el dibujo artístico y técnico, también hace hincapié en el enfoque holístico y en el desarrollo de propuestas desde la interdisciplinariedad y la transversalidad. Partiendo del planteamiento de Eisner de "artisticidad, su diálogo se traduce en una hibridación entre dibujo técnico y artístico en el que, explorando los límites de los lenguajes y sus relaciones, se generan nuevos aprendizajes.

Por último Remigi Morant y Ricard Huerta en el artículo Intercambio tecnológico. Intereses del nuevo profesorado de educación secundaria, nos aproximan a través de su investigación a los indicadores corporativos del futuro profesorado de las especialidades de Dibujo y Música. Cuestiones como el uso de las tecnologías o su percepción como colectivo son analizadas en el mismo.

Dentro del capítulo de experiencias docentes el artículo de Lucía Hervás, Iniciativas didácticas para el acercamiento en los centros educativos de propuestas sobre arte objetual, relata un proyecto llevado a cabo en diversos institutos. Partiendo de la obra de artistas como Carmen Calvo, Chema Madoz o Joan Brossa, se realizan actividades que permiten acercar el arte contemporáneo a los alumnos, proporcionando de este modo una visión más amplia del arte e iniciando a partir de las mismas una reflexión sobre las ideas preconcebidas que tienen sobre el arte actual.

En el mismo sentido de incorporación de prácticas artísticas contemporáneas, el artículo de Carolina Margarita Corbetta, Escuela, grafitis y cultura visual en la era digital, destaca la necesidad de acercar las prácticas docentes a los entornos cotidianos del alumnado, en este caso haciendo uso de un repertorio de imágenes basadas en el arte urbano y en el uso de las tecnologías. Se plantea de ese modo una ruptura de los límites de la escuela, acercando el espacio público y el escolar. Sus presupuestos conectarían con la educación artística y la cultura visual, en el que podríamos situar los artículos siguientes.

El artículo de Alberto Lombo, El graffiti fálico. Un análisis de ejemplos encontrados en la ciudad de Zaragoza, analiza las formas de expresión que emite el entorno urbano. Su estudio iconográfico arranca del origen histórico de esta representación simbólica y evidencia cómo la ciudad puede ser soporte de las expresiones populares. 
Acercándonos al contexto escolar, Luis Hernán Errázuriz, en el texto Propuestas para mejorar la estética cotidiana del entorno escolar, nos sitúa en el currículo oculto visual de los centros escolares. Su estudio teórico y de campo concluye con once propuestas que pueden contribuir a mejorar la estética cotidiana del entorno escolar.

En el ámbito de la educación artística y la educación patrimonial, Carmen Gómez, en El origen de los procesos de patrimonialización, realiza una reflexión sobre la acción educativa como detonante y catalizador de los procesos de patrimonialización, tanto a nivel individual, desde la vinculación afectiva con los objetos, como a nivel colectivo, en la conformación de comunidades.

El artículo de Julia Rocha, Discursos sobre el campo. Una inmersión en el Servicio Educativo del Museu de Arte Contemporânea de la Fundação de Serralves, aborda un análisis del "Proyeto anual de Escuelas", desarrollado por el Servicio Educativo del Museu de Arte Contemporânea de la Fundação de Serralves para la comunidad escolar. En este estudio se destaca la evaluación que desde el Servicio Educativo se realiza de su labor, así como de la necesaria conexión entre museo y escuela.

En relación con el diseño de los currículos escolares, Macarena Pérez, en $L a$ expresión musical al servicio de la integración del conocimiento: Música en Educación Secundaria y currículum en red, plantea un diseño de currículum integrado o currrículum en red. Mediante el diseño de retículas y la interconexión de conceptos se incide en la interdisciplinariedad y cómo éste puede incidir en el desarrollo de las competencias básicas.

Las potencialidades y los nuevos debates que plantea la Red se abordan en los siguientes artículos. En Creación de redes globales a través de acciones locales: Encuentros alternativos en la Educación Artística, Teresa Torres y Ana $\mathrm{M}^{\mathrm{a}}$ Barbero destacan el potencial de las redes para el trabajo colaborativo, analizando diferentes proyectos llevados a través de diversas redes y su contribución a la generación de un conocimiento colectivo.

Por último la entrevista realizada por Pau Farell al artista Joan Fontcuberta ( $A l$ otro lado del espejo de Joan Fontcuberta), permite cerrar este recorrido con una reflexión sobre los límites de la realidad y la ficción tratando cuestiones clave como la autoría, la identidad y el exhibicionismo.

A modo de síntesis podemos considerar que, aún desde diversas vertientes, encontramos múltiples conexiones entre los textos que presentamos en este número y que nos remiten a: interdisciplinariedad, transversalidad, entornos cotidianos, acercamiento al arte contemporáneo, cooperación, colaboración, trabajo por proyectos, afectividad e identidades. Consideramos también que a partir de los 
mismos se abren nuevas reflexiones e investigaciones que pueden contribuir a reivindicar el papel crucial que la Educación Artística desempeña en la formación integral del ciudadano libre del siglo XXI.

\section{Referencias bibliográficas}

Alderoqui, S. y Pedersoli, C. (2011). La educación en los museos. De los objetos a los visitantes. Buenos Aires: Paidós.

Augustowsky, G. (2012): El arte en la enseñanza. Buenos Aires: Paidós.

Duncum, P. (2002). Visual Culture Art Education: Why, What and How, International Journal of Art and Design Education, 21 (1), pp. 14-23.

Irwin, R. \& O’Donoghue, D. (2011). Encountering Pedagogy through Relational Art Practices, International Journal of Art and Design Education, 31 (3), pp. 221236.

Huerta, R. (2010). Un proyecto de investigación en educación artística: aspectos identitarios de las maestras chilenas, Pulso, 33, pp. 31-59.

Huerta, R. (2014a). Education on Sexual Diversity through Cinema, en Procedia - Social and Behavioral Sciences, 132, pp. 371-376. http://dx.doi.org/10.1016/j. sbspro.2014.04.324

Huerta, R. (2014b). La supuestamente ilegible escritura de médico como artefacto visual y estímulo creativo para la educación artística, Arte, Individuo y Sociedad, 26 (2), pp. 317-336. http://dx.doi.org/10.5209/rev_ARIS.2014.v26.n2.41883

Parker, T. (2009). Continuing the Journey - the Artist-Teacher MA as a Catalyst for Critical Reflection, International Journal of Art and Design Education, 28 (3), pp. 279-286.

Read, H. (1982). Educación por el arte. Barcelona: Paidós. 